\title{
Estudo farmacognóstico e atividade in vitro sobre a coagulação sanguínea e agregação plaquetária das folhas de Passiflora nitida Kunth (Passifloraceae)
}

\author{
Maria José de CARVALHO ${ }^{1}$, Tatiana do Nascimento PEDROSA², Fernanda GUILHON-SIMPLICIO ${ }^{3}$, Cecília \\ Verônica NUNEZ ${ }^{4}$, Débora Teixeira OHANA ${ }^{5}$, Maria de Meneses PEREIRA ${ }^{6}$, Emerson Silva LIMA
}

\section{RESUMO}

O gênero Passiflora (Passifloraceae) é utilizado principalmente para tratar doenças do SNC e cardiovasculares. A espécie Passiflora nitida Kunth é comumente conhecida como "maracujá-do-mato". A literatura relata o consumo in natura dos frutos desta espécie pela população local para distúrbios gastrointestinais. Considerando o potencial farmacológico do gênero, este trabalho teve por objetivo realizar estudo de caracterizaçáo fitoquímica desta espécie e estudar os efeitos dos extratos aquoso (EA), etanólico (EE) e hexânico $(\mathrm{EH})$ de suas folhas sobre a coagulação sanguínea e agregação plaquetária. Para a caracterização fitoquímica foram realizados testes de cromatografia em camada delgada e ressonância magnética nuclear. O efeito dos extratos sobre a coagulação foi avaliado pelos testes de tempo de protrombina (TP) e tempo de tromboplastina parcial ativada (TTPa). O efeito sobre a agregação plaquetária foi avaliado em plasma rico em plaquetas por método espectrofotométrico, usando adenosina difosfato (ADP) e adrenalina (ADR) como indutores da agregação. Os extratos EA, EE e EH apresentaram atividade coagulante pelo teste do TP e o EE apresentou atividade anticoagulante para o TTPa. Quando induzidos por ADP, os extratos EA, EE e EH apresentaram valores de concentração inibitória 50\% $\left(\mathrm{CI}_{50} \mu \mathrm{g} / \mathrm{mL}\right)$ de 450,5 \pm 50,7; 511,2 \pm 35,5 e $394,4 \pm 8,9$, respectivamente, e quando induzidos por ADR apresentaram valores de 438,7 \pm 5,2; 21,0 $\pm 1,9$ e 546,9 \pm 49,9, respectivamente. $\mathrm{O} E \mathrm{E}$ apresentou atividade inibitória sobre a agregação. A caracterização fitoquímica foi sugestiva da presença de flavonóides e cumarinas, aos quais podem ser atribuídos, em parte, os efeitos biológicos estudados.

PALAVRAS-CHAVE: Passiflora nitida, Coagulação sanguínea, Agregação plaquetária.

\section{Pharmacognostic study and in vitro activity on blood coagulation and platelet aggregation of leaves of Passiflora nitida Kunth (Passifloraceae)}

\section{ABSTRACT}

The Passiflora genus (Passifloraceae) is mainly used to treat CNS and cardiovascular diseases. The Passiflora nitida Kunth species is commonly known as "maracujá-do-mato". The literature reports the in natura consumption of fruits of this species by the local population for gastrointestinal disorders. Considering the pharmacological potential of the genus, this work aimed to carry out study of phytochemical characterization of this species and study the effects of the aqueous (AE), ethanol (EE) and hexane (HE) extracts from its leaves on blood coagulation and platelet aggregation. Thin-layer chromatography and nuclear magnetic resonance were carried out for the phytochemical characterization. The effect of the extracts on the coagulation was evaluated by prothrombin time (PT) and activated partial thromboplastin time (aPTT) tests. The effect on the platelet aggregation was evaluated in platelet-rich plasma by spectrophotometric method, using adenosine diphosphate (ADP) and adrenaline (ADR) as inducers of aggregation. The AE, EE and HE extracts showed coagulant activity by the PT test, and the EE showed anticoagulant activity by the aPTT. When induced by ADP, the AE, EE and HE extracts showed $50 \%$ inhibitory concentration values $\left(\mathrm{IC}_{50}, \mu \mathrm{g} / \mathrm{mL}\right)$ of $450.5 \pm 50.7,511.2 \pm 35.5$ and $394.4 \pm 8.9$, respectively, and when induced by ADR showed values of $438.7 \pm 5.2,21.0 \pm 1.9$ and 546.9 \pm 49.9 , respectively. The EE showed inhibitory effect on the aggregation. The phytochemical characterization was suggestive of the presence of flavonoids and coumarins, which can be attributed in part to the biological effects studied.

KEYWORDS: Passiflora nitida, Blood coagulation, Platelet aggregation.

\footnotetext{
1 Universidade Federal do Amazonas. E-mail: mjose_decarvalho@hotmail.com

2 Universidade Federal do Amazonas. E-mail: tpedrosa3@hotmail.com

3 Universidade Federal do Amazonas. E-mail: fernanda_guilhon@yahoo.com.br

${ }^{4}$ Instituto Nacional de Pesquisas da Amazônia. E-mail: cecilia@inpa.gov.br

5 Universidade Federal do Amazonas. E-mail: deboraohana@yahoo.com.br

6 Universidade Federal do Amazonas. E-mail: mariameneses@ufam.edu.br

Universidade Federal do Amazonas. E-mail: eslima@ufam.edu.br
} 


\section{INTRODUÇÃO}

As plantas medicinais são empregadas na prevenção, tratamento e cura de doenças como primeiro recurso medicinal desde a antiguidade, tendo representado a base da terapêutica durante muitos anos (Garcia et al., 1995; Silva e CechinelFilho, 2002).

Passiflora nitida Kunth (Passifloraceae) é uma espécie da flora amazônica conhecida popularmente como "maracujá-domato”, que cresce espontaneamente na vegetação secundária, beira de rios e estradas e seus frutos são consumidos in natura e usados na medicina tradicional para tratar distúrbios gastrointestinais. Esta espécie está dispersa por todo o norte da América do Sul e tem sido descrita como antioxidante e como antimicrobiana, sendo outras propriedades terapêuticas praticamente desconhecidas (Hopkins e Souza, 1999; Moraes et al., 2002; Bendini et al., 2006; INPI, 2006; IPGRI e CIRAD, 2006; Junqueira et al., 2007). Estudos já comprovaram propriedades farmacológicas em outras espécies do gênero Passiflora, tais como ansiolítica, sedativa, antiinflamatória, antinociceptiva e antihipertensiva, as quais foram relacionadas principalmente à presença de compostos flavonoídicos (Soulimani et al., 1997; Di Stasi et al., 2002; Dhawan e Sharma, 2002, 2003; Dhawan et al., 2004; Benincá et al., 2007).

Distúrbios da hemostasia são frequentes na prática médica e responsáveis por inúmeras condições patológicas (Pereira e Bogliolo, 2006). No tratamento e prevenção das doenças cardiovasculares (DCV), que representam a primeira causa de mortalidade no Brasil, a manutenção da hemostasia, principalmente quanto à agregação plaquetária é de importância fundamental, uma vez que seu descontrole é fator crítico na trombose arterial, fato esse que pode causar desordens com risco de vida, tais como ataques cardíacos, angina instável e reoclusão após angioplastia (Kim e Lee, 2006).

Estudos recentes sugerem efeitos benéficos dos flavonóis, flavonas e lignanas sobre DCV e têm mostrado que algumas flavonas atuam como um agente antiagregante, inibindo a atividade da enzima COX de plaquetas humanas (Silva et al., 2002; Arts e Hollman, 2005). Portanto, esforços consideráveis têm sido direcionados em torno da descoberta e desenvolvimento de produtos naturais com atividade coagulante/anticoagulante e antiagregante plaquetária, entre outros efeitos terapêuticos, mais eficazes e/ou com menos efeitos colaterais, como alternativa para os sintéticos e clássicos agentes terapêuticos.

Tendo em vista o potencial terapêutico do gênero Passiflora e o fato de não haver relatos de trabalhos referentes ao seu efeito sobre a coagulação sanguínea e agregação plaquetária e de haver pouca informaçáo sobre a espécie em estudo, tipicamente amazônica, foram realizados testes visando elucidar possíveis efeitos da $P$. nitida sobre estas atividades, bem como fitoquímicos.

\section{MATERIAL E MÉTODOS}

\section{REAGENTES}

$\mathrm{Na}$ cromatografia em camada delgada (CCD) foram utilizados ácidos, bases, sais, solventes e reagentes de pureza analítica (P.A.). Na espectrometria de ressonância magnética nuclear de ${ }^{1} \mathrm{H}\left(\mathrm{RMN} \mathrm{de}{ }^{1} \mathrm{H}\right)$ foram utilizados água deuterada $\left(\mathrm{D}_{2} \mathrm{O}\right)$ e tetrametilsilano (TMS). No teste farmacológico de coagulação sanguínea (TP e TTPa) foram utilizados os kits comerciais HemoStat Thromboplastin-SI e HemoStat aPTTEL (Human; Wiesbaden, Hessen, Alemanha), enquanto que no teste de agregação plaquetária, os indutores adenosina difosfato (Sigma-Aldrich ${ }^{\oplus}$, Saint Louis, Missouri, EUA) e adrenalina (Chrono-log, Havertown, Pennsylvania, EUA).

\section{OBTENÇÃO DA MATÉRIA-PRIMA VEGETAL (MPV)}

As folhas de $P$. nitida foram coletadas na comunidade ribeirinha do Careiro-Castanho, município do Careiro no Estado do Amazonas, nos meses de janeiro e fevereiro de 2007. A espécie do gênero Passiflora foi taxonomicamente identificada com o auxílio do Herbário do Instituto Nacional de Pesquisas da Amazônia (INPA), sendo comparada com a exsicata de número 209547 da coleção armazenada.

As folhas de $P$. nitida foram selecionadas, devidamente limpas e secas em estufa com circulaçáo interna de ar a $40^{\circ} \mathrm{C}$ por um período de sete dias, até que apresentassem aspecto quebradiço. Posteriormente, a amostra foi pulverizada em moinho de facas, constituindo, então, a matéria-prima vegetal (MPV) que foi utilizada.

\section{OBTENÇÃO DOS EXTRATOS}

Os extratos brutos aquoso (EA), etanólico (EE) e hexânico (EH) de P. nitida foram obtidos a partir de três alíquotas de $200 \mathrm{~g}$ de MPV, através do método de maceração estática com $1000 \mathrm{~mL}$ de solvente respectivo a cada tipo de extraçáo, à temperatura ambiente, por um período de sete dias.

Após filtraçôes, o solvente dos extratos EE e EH foram evaporados em rotaevaporador, sob pressão reduzida e baixa temperatura, enquanto o extrato EA foi liofilizado, gerando os extratos brutos da planta, que foram utilizados nos ensaios biológicos.

\section{TESTES FITOQUÍMICOS}

\section{Cromatografia em camada delgada (CCD)}

A CCD em sílica gel foi realizada com pequenas alíquotas dos extratos brutos EA, EE e EH das folhas de $P$. nitida solubilizadas em quantidade suficiente de metanol. Foram utilizados como sistemas de eluição o acetato de etila/acetona 
2:3, metanol puro e butanol/ácido acético/água (BAW) 4:0,5:0,5.

Após a saturação das cubas com os eluentes, as cromatofolhas de alumínio impregnadas com sílica gel, contendo os extratos EA, EE e EH recém-aplicados, foram eluídas. Após eluição, as placas foram reveladas com reagentes específicos para detecção de substâncias sequestradoras de radicais livres e de compostos fenólicos (Zweig e Sherma, 1987; Zuanazzi e Montanha, 2007). As leituras foram efetuadas em câmara escura sob luz branca e UV nos comprimentos de onda $\lambda_{254}$ e $\lambda_{365} \mathrm{~nm}$.

Espectrometria de ressonância magnética nuclear de ${ }^{1} \mathrm{H}\left(\mathrm{RMN}\right.$ de $\left.{ }^{1} \mathrm{H}\right)$

O extrato bruto EE foi submetido à análise por ressonância magnética nuclear (RMN) de ${ }^{1} \mathrm{H}$, sendo uma alíquota da amostra dissolvida em $600 \mu \mathrm{L}$ de água deuterada $\left(\mathrm{D}_{2} \mathrm{O}\right)$. Todos os espectros foram obtidos na frequência de $500 \mathrm{MHz}$ em espectrômetro de RMN (Varian, modelo Inova 500, Palo Alto, Canada, EUA). Os deslocamentos químicos foram obtidos em partes por milhão (unidades de $\delta$ ), com relação ao tetrametilsilano (TMS), tomado como referência interna.

\section{TESTES BIOLÓGICOS}

\section{Coagulação sanguínea}

Os efeitos dos extratos EA, EE e EH das folhas de P. nitida sobre a coagulaçáo sanguínea foram avaliados em plasma pobre em plaquetas (PPP) pelo método de Brown (1988) modificado por Osoniyi e Onajobi (2003). Foram realizados os testes de tempo de protrombina (TP) e tempo de tromboplastina parcial ativada (TTPa), utilizando-se coagulômetro (Teco, modelo Coatrom M1, Alemanha) e os kits comerciais HemoStat Thromboplastin-SI e HemoStat aPTT-EL (Human, Alemanha). Para o TP, foram adicionados $96 \mu \mathrm{L}$ de reagente Thromboplastin-SI a $50 \mu \mathrm{L}$ de PPP previamente incubado com $4 \mu \mathrm{L}$ dos respectivos extratos nas concentraçóes de 50 , 25 e $12,5 \mathrm{mg} / \mathrm{mL}$. Para os testes de TTPa, foram adicionados $36 \mu \mathrm{L}$ de $\mathrm{CaCl}_{2}$ a $30 \mu \mathrm{L}$ de PPP previamente incubado em banho-maria com $30 \mu \mathrm{L}$ de reagente HemoStat aPTT-EL e $4 \mu \mathrm{L}$ dos respectivos extratos. Os valores dos testes obtidos a partir da adição de extrato ao PPP foram comparados com os resultados dos testes com os solventes, dimetilsulfóxido (DMSO) para os extratos EE e EH e água Milli-Q para EA.

Agregação plaquetária

Os efeitos dos extratos EA, EE e EH sobre a agregação plaquetária foram avaliados em plasma rico em plaquetas (PRP) humano pelo método de Born e Cross (1963) com adaptaçôes, utilizando-se um agregômetro de plaquetas estabilizado na temperatura de $37^{\circ} \mathrm{C}$ (Qualiterm, modelo PA-04, Brasil). O PRP foi submetido aos agentes indutores de agregação plaquetária adenosina difosfato (ADP) e adrenalina
(ADR), ambos na concentração de $10 \mu \mathrm{mol} / \mathrm{L}$, e em seguida, uma curva de agregaçáo foi plotada. O PPP contendo o diluente dos extratos foi utilizado como controle dos testes. Brevemente, $485 \mu \mathrm{L}$ de PRP foram individualmente incubadas por 2 minutos com $5 \mu \mathrm{L}$ dos extratos nas concentraçóes de 50,25 e $12,5 \mathrm{mg} / \mathrm{mL}$, antes da adição do agente indutor e o efeito sobre a curva de agregaçáo foi monitorado por 5 min. A percentagem de inibição foi dada pela fórmula: 100 (agregação da amostra / agregação do controle) x 100. O efeito inibitório dos extratos foi comparado com aqueles do ácido acetilsalicílico (AAS), por meio do cálculo da $\mathrm{CI}_{50}$, utilizada como parâmetro para a avaliação da atividade antiagregante plaquetária (MEKHFI et al., 2004; KIM; LEE, 2006).

Os procedimentos seguidos estiveram de acordo com os padrōes éticos do Comitê de Ética em Pesquisa da Universidade Federal do Amazonas - CEP/UFAM (processo número 038/2006).

\section{ANÁLISE ESTATÍSTICA DOS DADOS}

Os resultados obtidos foram analisados com o programa SigmaStat (versão 3.5 para Windows; Systat Software, San Jose, California, EUA), expressos como média \pm EPM e apresentados na forma gráfica por utilização do programa Excel (Microsoft ${ }^{\oplus}$; Seattle, Washington, EUA). A normalidade foi verificada através do teste de Kolmogorov Smirnov e para múltiplas comparaçóes, foi utilizada a análise de variância com um único fator (ANOVA one way), complementada pelo teste de Tukey. O nível de significância considerado para os testes foi $\mathrm{P}<0,05$. A determinação da $\mathrm{CI}_{50}$ do efeito sobre a agregação plaquetária foi realizada pelo método de regressão linear, usando o programa Origin $^{\oplus}$ (versão 8.0; Micronal Software, Northampton, Massachusetts, EUA).

\section{RESULTADOS E DISCUSSÃO}

$\mathrm{Na}$ CCD, os extratos brutos EA, EE e EH das folhas de P. nitida foram positivos para substâncias sequestradoras de radicais livres e compostos fenólicos (Zweig e Sherma, 1987; Zuanazzi e Montanha, 2007).

Tabela 1 - Detecção da presença de compostos fenólicos e de substâncias sequestradoras de radical livre nos extratos brutos das folhas de Passiflora nitida Kunth por reagentes específicos em CCD (Fonte: Zweig e Sherma, 1987; Zuanazzi e Montanha, 2007)

\begin{tabular}{llll}
\hline Reagente & $\mathrm{EA}^{1}$ & $\mathrm{EE}^{2}$ & $\mathrm{EH}^{3}$ \\
\hline $\mathrm{DPPH}(0,157 \mathrm{mg} / \mathrm{mL})$ & ++ & ++ & + \\
$\mathrm{AlCl}_{3} 1 \%$ alcoólico & + & + & + \\
$\mathrm{NaOH}_{2} \%, \mathrm{NH}_{4} \mathrm{OH}, \mathrm{Na}_{2} \mathrm{CO}_{3} 0,2 \%$ & + & + & + \\
$\mathrm{FeCl}_{3} 1 \%$ alcoólico & + & + & + \\
Folin-Ciocalteau & + & + & + \\
\hline
\end{tabular}

1: EA = extrato aquoso das folhas de Passiflora nitida Kunth;

2: $\mathrm{EE}=$ extrato etanólico das folhas de P. nitida Kunth;

3: $\mathrm{EH}=$ extrato hexânico das folhas de $P$. nitida Kunth.

$+=$ positivo; $++=$ branco, significando intensa atividade sequestradora de radicais livres. 
Uma vez que o extrato havia apresentado atividade sequestradora de radicais livres e revelou também a presença de substâncias aromáticas, o mesmo foi então submetido à análise por $\mathrm{RMN}$, usando $\mathrm{D}_{2} \mathrm{O}$ (Figura 1). $\mathrm{O}$ sinal em $-4,6$ ppm é atribuído a hidrogênio ligado ao carbono anomérico de açúcar, como o $J$ (constante de acoplamento) $=8 \mathrm{~Hz}$, referente à configuraçáo $\beta$. Os sinais entre 5-5,2 e 5,9-6,2 ppm são atribuídos a ligaçôes duplas isoladas. Os sinais entre 6,7-6,9 e 7,6-8,3 ppm são atribuídos a compostos aromáticos (Silverstein et al., 2007).

\section{A}

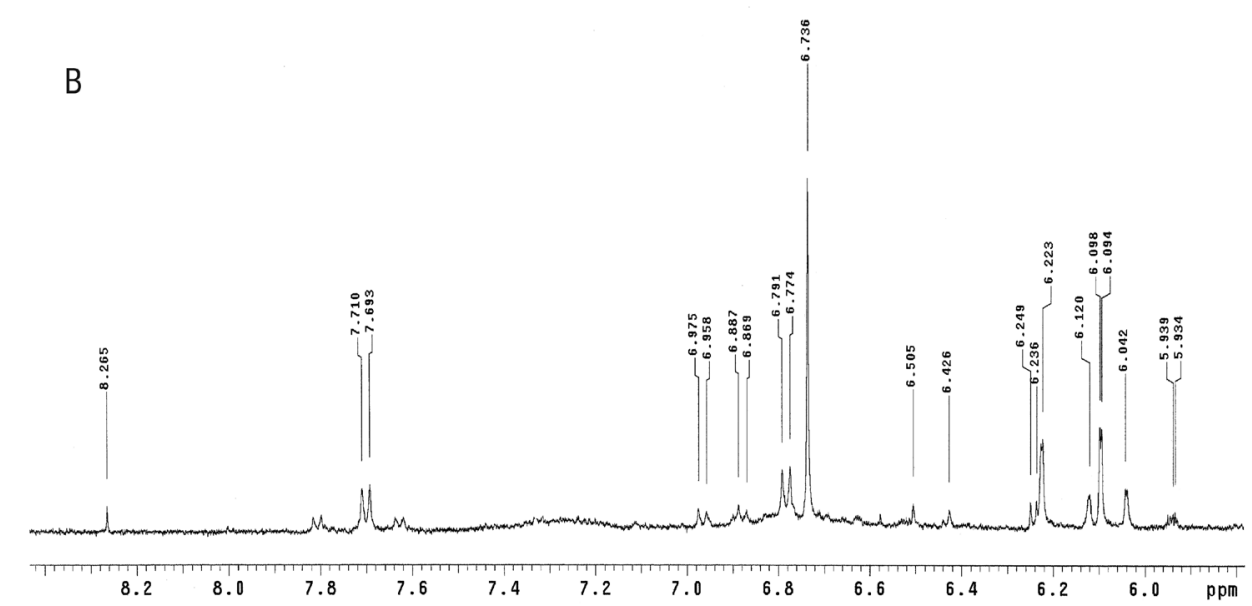

Figura 1 - Expansões nas regiões entre: (A) $\delta_{H} 4,46-5,68$ e (B) $\delta_{H} 5,8-8,4$ do espectro de RMN de ${ }^{1} \mathrm{H}$ do extrato bruto etanólico (EE) das folhas de Passiflora nitida Kunth $\left(\mathrm{D}_{2} \mathrm{O}, 500 \mathrm{MHz}\right)$. 
Como apresentado na Tabela 2 quando examinados pelo TP, os extratos brutos EA, EE e EH das folhas de $P$. nitida apresentaram atividade coagulante estatisticamente significativa $(\mathrm{P}<0,05)$. Os dados sugerem que essa atividade seja devida à interferência dos extratos na via extrínseca da coagulaçáa (fator VII). O extrato EE, por sua vez, apresentou atividade anticoagulante $(\mathrm{P}<0,05)$ no TTPa, o que sugere que ele inibe um ou mais fatores da via intrínseca da coagulaçáo (fatores VIII, IX, XI, XII). A atividade do extrato EE sobre a coagulação sanguínea está em consonância com as propriedades farmacológicas de inibição da coagulação, relatadas na literatura para flavonóides e cumarinas (Kuster e Rocha, 2007; Zuanazzi e Montanha, 2007), sugestivamente presentes na caracterização fitoquímica realizada neste trabalho.

Uma possível explicação para o comportamento do extrato EE, estaria no fato de o mesmo fator agir como um procoagulante e como um anticoagulante sob condiçóes diferentes. Por exemplo, foi comprovado que a trombina age como um procoagulante ao clivar o fibrinogênio, promovendo a formação de um coágulo de fibrina. Mas, age como um anticoagulante ao ativar a proteína $\mathrm{C}$ na presença do co-fator trombomodulina (Osoniyi e Onajobi, 2003; Kumar et al., 2005; Pereira e Bogliolo, 2006). A atividade coagulante dos extratos EA, EE e EH confirma, em parte, o uso popular do gênero Passiflora contra picadas de cobra (Dhawan et al., 2004).

Os extratos também foram testados quanto ao efeito sobre a agregação plaquetária. Os extratos EA, EE e EH apresentaram baixa atividade sobre a inibição da agregação induzida pelos agentes indutores de agregação plaquetária ADP e ADR (Tabela 3). Os valores de $\mathrm{CI}_{50}$ apresentaram uma atividade não seletiva por agentes indutores de agregação (Tabela 4). A espécie estudada apresentou efeito inibitório
Tabela 2 - Efeitos de extratos brutos das folhas de Passiflora nitida Kunth sobre a coagulação sanguínea

\begin{tabular}{|c|c|c|c|c|}
\hline \multirow{2}{*}{ Amostra } & \multicolumn{2}{|c|}{ Concentração (mg/mL) } & \multirow{2}{*}{$\mathrm{TP}(\mathrm{s})$} & \multirow{2}{*}{$\operatorname{TTPa}(\mathrm{s})$} \\
\hline & TP & TTPa & & \\
\hline Controle & $\begin{array}{l}\text { Água } \\
\text { Milli-Q }\end{array}$ & & $16,9 \pm 0,2$ & $38,0 \pm 4,0$ \\
\hline \multirow[t]{3}{*}{$E A^{1}$} & 1,3 & 2 & $15,9 \pm 0,1$ & $34,9 \pm 2,4$ \\
\hline & 0,7 & 1 & $15,3 \pm 0,2^{*}$ & $38,1 \pm 1,0$ \\
\hline & 0,3 & 0,5 & $16,5 \pm 0,3$ & $39,4 \pm 5,2$ \\
\hline Controle & DMSO & & $19,5 \pm 0,5$ & $59,8 \pm 1,9$ \\
\hline \multirow[t]{3}{*}{$\mathrm{EE}^{2}$} & 1,3 & 2 & $7,8 \pm 0,7^{\star}$ & $65,2 \pm 2,6$ \\
\hline & 0,7 & 1 & $19,6 \pm 0,1$ & $70,4 \pm 3,0$ * \\
\hline & 0,3 & 0,5 & $19,6 \pm 0,4$ & $61,3 \pm 1,2$ \\
\hline \multirow[t]{3}{*}{$\mathrm{EH}^{3}$} & 1,3 & 2 & $8,6 \pm 0,7^{\star}$ & $53,5 \pm 1,1$ \\
\hline & 0,7 & 1 & $19,2 \pm 0,2$ & $51,8 \pm 1,1$ \\
\hline & 0,3 & 0,5 & $20,0 \pm 0,3$ & $52,9 \pm 0,5$ \\
\hline
\end{tabular}

Os resultados foram expressos como média \pm erro padrão da média (EPM) com $n=2-7$.

${ }^{*} P<0,05$ comparado com o controle (análise de variância - ANOVA, complementada pelo teste de Tukey)

1: EA = extrato aquoso das folhas de Passiflora nitida Kunth;

2: $\mathrm{EE}=$ extrato etanólico das folhas de $P$. nitida Kunth;

3 : $\mathrm{EH}=$ extrato hexânico das folhas de $P$. nitida Kunth.

mais acentuado quanto ao extrato $\mathrm{EE}$ induzido por ADR $\left(\mathrm{CI}_{50}=21,0 \pm 1,9 \mu \mathrm{g} / \mathrm{mL}\right)$, quando comparado ao padráo AAS $\left(\mathrm{CI}_{50}=11,7 \pm 0,3 \mu \mathrm{g} / \mathrm{mL}\right)$.

A atividade antiagregante da $P$. nitida pode ser atribuída, em parte, à presença de flavonóides e cumarinas, sugestivamente presentes na caracterização fitoquímica realizada para esta espécie. Segundo a literatura, substâncias dessas classes podem inibir a agregação plaquetária (Kuster e Rocha, 2007; Zuanazzi e Montanha, 2007).

A análise dos extratos brutos EA, EE e EH das folhas de $P$. nitida sugerem a presença de constituintes ativos com potencialidades para fins terapêuticos, como demonstrado nos

Tabela 3 - Efeitos de extratos brutos das folhas de Passiflora nitida Kunth sobre a agregação plaquetária induzida por ADP e ADR

\begin{tabular}{|c|c|c|c|c|c|}
\hline \multirow{2}{*}{ Amostra } & \multirow{2}{*}{ Concentração ( $\mu \mathrm{g} / \mathrm{mL})$} & \multicolumn{2}{|c|}{ ADP $(10 \mu \mathrm{mol} / \mathrm{L})$} & \multicolumn{2}{|c|}{ ADR $(10 \mu \mathrm{mol} / \mathrm{L})$} \\
\hline & & Agregação (\%) & Inibição (\%) & Agregação (\%) & Inibição (\%) \\
\hline Controle & Água Milli-Q & $62,2 \pm 1,8$ & - & $84,7 \pm 5,8$ & - \\
\hline \multirow[t]{3}{*}{$E A^{1}$} & 500 & n.d. & n.d. & $25,5 \pm 0,5^{*}$ & $69,9 \pm 0,6$ \\
\hline & 250 & $48,3 \pm 2,1^{\star}$ & $22,3 \pm 3,5$ & $73,0 \pm 0,0$ & $13,8 \pm 0,0$ \\
\hline & 125 & $55,5 \pm 1,5$ & $10,8 \pm 2,4$ & $84,5 \pm 0,5$ & $0,2 \pm 0,6$ \\
\hline Controle & DMSO & $69,0 \pm 1,1$ & - & $67,0 \pm 4,0$ & - \\
\hline \multirow[t]{3}{*}{$\mathrm{EE}^{2}$} & 500 & $36,3 \pm 3,2^{*}$ & $47,3 \pm 4,6$ & $41,0 \pm 4,0^{*}$ & $38,8 \pm 5,9$ \\
\hline & 250 & $36,5 \pm 1,5^{\star}$ & $47,1 \pm 2,2$ & $46,5 \pm 2,5^{*}$ & $30,6 \pm 3,7$ \\
\hline & 125 & $47,0 \pm 2,0^{\star}$ & $31,9 \pm 2,9$ & $51,5 \pm 0,5$ & $23,1 \pm 0,8$ \\
\hline Controle & DMSO & $72,0 \pm 0,4$ & - & $57,0 \pm 4,0$ & - \\
\hline \multirow[t]{3}{*}{$\mathrm{EH}^{3}$} & 500 & $31,5 \pm 1,5^{\star}$ & $56,3 \pm 2,0$ & $32,5 \pm 1,5^{\star}$ & $42,9 \pm 2,6$ \\
\hline & 250 & $38,5 \pm 0,5^{\star}$ & $46,5 \pm 0,7$ & $42,5 \pm 1,5$ & $25,4 \pm 2,6$ \\
\hline & 125 & $56,5 \pm 0,5^{\star}$ & $21,5 \pm 0,7$ & $55,5 \pm 1,5$ & $1,8 \pm 2,6$ \\
\hline
\end{tabular}


Tabela 4 - Valores de $\mathrm{Cl}_{50}$ dos extratos brutos das folhas de Passiflora nitida Kunth na agregação plaquetária induzida pelos agentes indutores de agregação plaquetária ADP e ADR

\begin{tabular}{lcc}
\hline \multirow{2}{*}{ Amostra } & \multicolumn{2}{c}{$\mathrm{Cl}_{50}(\mu \mathrm{g} / \mathrm{mL})^{1}$} \\
\cline { 2 - 3 } & $\operatorname{ADP}(0,4 \mathrm{mg} / \mathrm{mL})$ & $\mathrm{ADR}(10 \mu \mathrm{mol} / \mathrm{L})$ \\
\hline $\mathrm{EA}^{2}$ & $450,5 \pm 50,7$ & $438,7 \pm 5,2$ \\
$\mathrm{EE}^{3}$ & $511,2 \pm 35,5$ & $21,0 \pm 1,9$ \\
EH $^{4}$ & $394,4 \pm 8,9$ & $546,9 \pm 49,9$ \\
AAS $^{5}$ & $21,5 \pm 1,4$ & $11,7 \pm 0,3$ \\
\hline
\end{tabular}

Cada valor representa a média \pm EPM para três experimentos $(125,250$ e $500 \mu \mathrm{g} / \mathrm{mL})$. 1: $\mathrm{Cl}_{5}=$ concentração $(\mu \mathrm{g} / \mathrm{mL})$ exigida para produzir $50 \%$ de inibicãao de agregacão plaguetária induzida pelos agentes indutores de agregacão plaquetária ADP e ADR:

2: EA = extrato aquoso das folhas de Passiflora nitida Kunth;

3 : $\mathrm{EE}=$ extrato etanólico das folhas de $P$. nitida Kunth;

4: $\mathrm{EH}=$ extrato hexânico das folhas de $P$. nitida Kunth

5: AAS = ácido acetilsalicílico

métodos empregados. Os resultados aqui obtidos demonstram outras possíveis aplicaçôes para esta espécie. A literatura reporta, essencialmente, as atividades gastrointestinal, antimicrobiana e antioxidante. No entanto, neste trabalho, foram observadas atividades sobre a coagulação sanguínea $e$ sobre a agregação plaquetária e, mesmo demonstrando baixa expressividade, é importante que sejam posteriormente melhor investigadas. Os mecanismos exatos das atividades terapêuticas dos extratos da $P$. nitida ainda são desconhecidos, devendo estes estudos serem acrescidos de fracionamento, isolamento e testes farmacológicos adicionais, visando à obtenção e caracterização estrutural de princípios ativos e elucidação dos mecanismos de ação das atividades estudadas.

\section{CONCLUSÃO}

$\mathrm{Na}$ caracterização fitoquímica das folhas de $P$. nitida, os compostos aromáticos destacaram-se como a classe majoritária de compostos químicos, à qual podem ser atribuídos, em parte, os efeitos biológicos estudados. Os extratos aquoso, etanólico e hexânico das folhas de $P$. nitida apresentam moderadas atividades coagulante e/ou anticoagulante. O extrato etanólico apresenta efeitos antiagregante plaquetário mais significativo. As folhas de $P$. nitida possuem constituintes ativos, com potencialidades para fins terapêuticos e são promissores para futuros estudos, visando, por exemplo, o desenvolvimento de formulaçōes fitoterápicas.

\section{AGRADECIMENTOS}

Ao MCT/CNPq/CT-Amazônia e à FAPEAM pelo apoio financeiro. À FCF/UFAM e ao CPPN/INPA pela disponibilização de laboratórios.

\section{BIBLIOGRAFIA CITADA}

Arts, I.C.W.; Hollman, P.C.H. 2005. Polyphenols and disease risk in epidemiologic studies. American Journal of Clinical Nutrition, 81: S317-S325.
Bendini, A.; Cerretani, L.; Pizzolante, L.; Toschi, T.G.; Guzzo, F.; Ceoldo, S.; Marconi, A.M.; Andreetta, F.; Levi, M. 2006. Phenol content related to antioxidant and antimicrobial activities of Passiflora spp. extracts. European Food Research Technology, 223: 102-109.

Benincá, J.P.; Montanher, A.B.; Zucolotto, S.M.; Schenkel, E.P.; Fröde, T.S. 2007. Evaluation of the anti-inflammatory efficacy of Passiflora edulis. Food Chemistry, 104: 1097-1105.

Born, G.V.R.; Cross, M.J. 1963. The aggregation of blood platelets. Journal of Physiology, 168: 178-195.

Dhawan, K.; Dhawan, S.; Sharma, A. 2004. Passiflora: a review update. Journal of Ethnopharmacology, 94: 1-23.

Dhawan, K.; Sharma, A. 2002. Prevention of chronic alcohol and nicotine-induced azospermia, sterility and decreased libido, by a novel tri-substituted benzoflavone moiety from Passiflora incarnata Linneaus in healthy male rats. Life Sciences, 71: 30593069.

Dhawan, K.; Sharma, A. 2003. Restoration of chronic- $\Delta^{9}-$ THCinduced decline in sexuality in male rats by a novel benzoflavone moiety from Passiflora incarnata Linn. British Journal of Pharmacology, 138: 117-120.

Di Stasi, L.C.; Hiruma-Lima, C.A.; Gonzales, F.G.; Portilho, W.G. 2002. Violales medicinais, p.177-199. In: Di Stasi, L.C.; Hiruma-Lima, C.A. Plantas medicinais na Amazônia e na Mata Atlântica. Editora da UNESP, SP, Brasil.

Garcia, E.S.; Silva, A.C.P; Gilbert, B.; Corrêa, C.B.V; Cavalheiro, M.V.S; Santos, R.R.; Tomassini, T. 1995. Biodiversidade: perspectivas e oportunidades. (www.bdt.org.br). Acesso: 26/04/06

Hopkins, M.J.G.; Souza, M.A.D. 1999. Passifloraceae, p. 299.In: Ribeiro, J.E.L.S.; Hopkins, M.J.G.; Vicentini, A.; Sothers, C.A.; Costa, M.A.S.; Brito, J.M.; Souza, M.A.D.; Martins, L.H.P.; Lohmann, L.G.; Assunção, P.A.C.L.; Pereira, E.C.; Silva, C.F.; Mesquita, M.R.; Procópio, L.C. Flora da Reserva Ducke: guia de identificação das plantas vasculares de uma floresta de terrafirme na Amazônia Central. Instituto Nacional de Pesquisas da Amazônia, Manaus, AM.

Instituto Nacional da Propriedade Industrial (INPI), 2006. Relação: frutas da Amazônia. (www.inpi.gov.br). Acesso : 09/05/06.

International Plant Genetic Resources Institute; Centre de Coopération Internationale en Recherche Agronomique pour le Développement (IPGRI; CIRAD), 2006. Passiflora nitida (H.B.K.) (Passifloraceae). (www.ciat.cgiar.org). Acesso: 26/04/06

Junqueira, K.P.; Faleiro, F.G.; Ramos, J.D.; Bellon, G.; Junqueira, N.T.V.; Braga, M.F. 2007. Variabilidade genética de acessos de maracujá-suspiro com base em marcadores moleculares. Revista Brasileira de Fruticultura, 29: 571-575.

Kim, M.; Lee, K. 2006. Antithrombotic activity of methanolic extract of Umbilicaria esculenta. Journal of Ethnopharmacology, 105: 342-345.

Kumar, V.; Abbas, A.K.; Fausto, N. (Eds). 2005. Inflamação aguda e crônica p. 49-89. In: Kumar, V.; Abbas, A.K.; Fausto, N. (Eds). 
Robbins \& Cotran, Patologia - bases patológicas das doenças. $7^{\mathrm{a}}$ ed., Elsevier, RJ, Brasil.

Kuster, R.M.; Rocha, L.M. 2007. Cumarinas, cromonas e xantonas, p. 537-556. In: Simóes, C.M.O.; Schenkel, E.P.; Gosman, G.; Mello, J.C.P.; Mentz, L.A.; Petrovick, P.R. (Org.). Farmacognosia: da planta ao medicamento. $6^{a}$ ed., Editora da UFRGS/Editora da UFSC, Porto Alegre/Florianópolis, RS/SC, Brasil.

Mekhfi, H.; El Haouari, M.; Legssyer, A.; Bnouham, M.; Aziz, M.; Atmani, F.; Remmal, A.; Ziyyat, A. 2004. Platelet antiaggregant property of some Moroccan medicinal plants. Journal of Ethnopharmacology, 94: 317-322.

Moraes, M.C.; Vieira, M.L.C.; Novaes, Q.S.; Rezende, J.A.M. 2002. Susceptibilidade de Passiflora nitida ao Passion fruit woodiness virus. Fitopatologia Brasileira, 27: 108.

Osoniyi, O.; Onajobi, F. 2003. Coagulant and anticoagulant activities in Jatropha curcas latex. Journal of Ethnopharmacology, 89: 101-105.

Pereira, F.E.L.; Bogliolo, L. 2006. Inflamaçôes, p. 130-174. In: Brasileiro-Filho, G. Bogliolo Patologia. $7^{\mathrm{a}}$ ed., Guanabara Koogan S.A, RJ, Brasil.

Silva, K.L.; Cechinel-Filho, V. 2002. Plantas do gênero Bauhinia: composição química e potencial farmacológico. Química Nova, 25: $449-454$.
Silva, R.R.; Oliveira, T.T.; Nagem, T.J.; Leão, M.A. 2002. Efeito de flavonóides no metabolismo do ácido araquidônico. Medicina, 35: 127-133.

Silverstein, R.M.; Webster, F.X.; Kiemle, D.J. 2007. Espectrometria de ressonância magnética de hidrogênio p. 123-197. In: Silverstein, R.M.; Webster, F.X.; Kiemle, D.J. Identificação espectrométrica de compostos orgânicos. $7^{\mathrm{a}}$ ed., LTC - Livros Técnicos e Científicos Editora S.A., RJ, Brasil.

Soulimani, R.; Younos, C.; Jarmouni, S.; Bousta, D.; Misslin, R.; Mortier, F. 1997. Behavioural effects of Passiflora incarnata L. and its indole alkaloid and flavonoid derivatives and maltol in the mouse. Journal of Ethnopharmacology, 57: 11-20.

Zuanazzi, J.A.S.; Montanha, J.A. 2007. Flavonóides, p. 577-614. In: Simóes, C.M.O.; Schenkel, E.P.; Gosman, G.; Mello, J.C.P; Mentz, L.A.; Petrovick, P.R. (Org.). Farmacognosia: da planta ao medicamento. $6^{\text {a }}$ ed., Editora da UFRGS/Editora da UFSC, Porto Alegre/Florianópolis, RS/SC, Brasil. .

Zweig, G.; Sherma, J. (Eds). 1987. CRC handbook of Chromatography: general data and principles. Vol. 2. CRF Press, Inc., Boca Raton, FL, USA. 173pp.

Recebido em 19/02/2009

Aceito em 18/06/2009 
